\title{
BONAVETURA DA BAGNOREGIO - UNA TEOLOGIA AFFETTIVA O SAPIENZIALE?
}

\author{
C T I R A D V.P O S P Í Š L
}

\section{ABSTRACT \\ Bonaveture of Bagnoregio: an Affective, or Sapiential Theology?}

The author in this study responds to the ambiguity about the concept of the theology of Bonaventure of Bagnoregio, specifically the expression that refers to "affective theology". Based on the source texts, he proves that Bonaventure's theology is sapiential, not just emotional and devotional. The term "affective" is necessary to understand as a synecdochical expression of the sapiential character of Bonaventure's theology. In the introduction to the Bonaventure's Commentary of the Sentences the author discovers a text in which Bonaventure portrays theology as wisdom in the middle of an imaginary cross with axes: intellect - affection, and contemplation - practice. This fact is up-to-date as the International Theological Commission, in one of its latest papers, encourages today's theologians not to forgive the sapiential dimension of their work.

Key words

Theology; Anthropology; Spirituality; Scholastic method in theology

DOI: $10.14712 / 23363398.2019 .9$

$\mathrm{N}_{\mathrm{ell}}$ la letteratura contemporanea dedicata all'interpretazione del pensiero bonaventuriano s'incontra spesso la valutazione, secondo la quale stiamo di fronte ad una teologia "affettiva". Il problema sta soprattutto nel vero significato della potenza affettiva nelle opere del nostro maestro. Chi non è informato, legge l'espressione appena menzionata in modo poco preciso e di conseguenza non prende sufficientemente in considerazione la componente intellettuale della 
teologia e della filosofia di Bonaventura. Non pochi poi sottovalutano il messaggio bonaventuriano, considerandolo come espressione di quasi pura devozionalità affettiva. Tutta l'esposizione analitica seguente viene, perciò, motivata dal desiderio di far fronte a questo malinteso poco felice e di chiarire la vera impostazione della teologia bonaventuriana.

Dato che l'affetto bonaventuriano, legato evidentemente ai sentimenti e alla volontà, di solito nella sua ricerca non viene staccato dall'intelletto ${ }^{1}$ si può facilmente intuire che rappresenta una componente movente dell'atto dell'intera mente umana, la quale abbraccia l'immagine psicologica della Trinità nella forma della memoria (il Padre), dell'intelletto (il Figlio) e della volontà in quanto la capacità dell'amare (lo Spirito Santo). Siccome la mente rappresenta la sede della sapienza, l'affetto bonaventuriano non dovrebbe essere preso come una semplice sentimentalità devota, ma piuttosto come la forza movente della sapienza. Nel primo paragrafo di questo studio perciò brevemente ricordiamo il significato della parola sapientia nel pensiero del Serafico. Nel secondo punto poi vogliamo analizzare un testo chiave, ${ }^{2}$ nel quale Bonaventura spiega la sua concezione della teologia. Nella conclusione mettiamo in luce l'attualità del modo di concepire la teologia secondo Bonaventura.

\section{La sapienza e la mente umana}

Il testo chiave che ci permette di penetrare più a fondo nel significato assai complesso della parola mens nell'accezione bonaventuriana si trova nel famoso scritto Itinerarium mentis in Deum. Tutto il terzo capitolo dell'Itinerarium è dedicato alla contemplazione di Dio - Trinità mediante la sua immagine, che s'identifica secondo Bonaventura con la mens. ${ }^{3}$ Il punto di partenza della riflessione consiste nell'affermazione che a Dio, che è lo spirito più perfetto, dobbiamo ascrivere le capacità della memoria, dell'intelletto e della volontà. Questa distinzione delle singole facoltà riflette in un certo senso il mistero della Trinità

1 Cf. Marianne Schlosser, "Affectio," in Dizionario Bonaventuriano. Filosofia - Teologia Spiritualità, ed. Ernesto Caroli (Padova: Editirici Francescane, 2008), 150-156. Il fatto che in questo dizionario manca la voce Sapientia, pare abbastanza strano.

2 Cf. Sent. I, prooem., q. III, resp.; I, p. 13; in S. Bonaventurae, Opera Omnia I-IX, Studio et cura PP. Collegii a S. Bonaventurae, I-X, Quaracchi - Florentia 1882-1902.

3 “... ubi ad modum candelabri relucet lux veritatis in facie nostrae mentis, in qua scilicet resplendet imago beatissimae Trinitatis." Itinerarium mentis in Deum, III, 1; Opera Omnia V, 303ab. 
immanente, cioè i rapporti tra il Padre, il Figlio e lo Spirito Santo. ${ }^{4}$ La natura divina, in quanto puro spirito, può essere denominata pure la mens perfetta. ${ }^{5}$ Le facoltà della mente umana, cioè la memoria, l'intelletto e la volontà, insieme riflettono il mistero della Santissima Trinità. ${ }^{6}$ La mente umana, perciò, in un certo senso corrisponde all'unica natura divina, ${ }^{7}$ la sua memoria rimanda alla persona del Padre, il suo intelletto alla persona del Figlio, la sua volontà, infine, alla persona dello Spirito Santo.

4 "Si igitur Deus perfectus est spiritus, habet memoriam, intelligentiam et voluntatem, habet et Verbum genitum et Amorem spiratum, qui necessario distinguuntur, cum unus ab altero producatur, non essentialiter, non accidentaliter, ergo personaliter." Itin., III, 5; V, 305ab.

Va ricordato, che l'identificazione dell'immagine di Dio con la mens deriva direttamente da Agostino e che la teologia cattolica odierna critica questa posizione, perché implica una certa inclinazione al dualismo. Cf. CTI, Comunione e servizio: La persona umana creata a immagine di Dio, documento dal 23 luglio 2004, n. 28, n. 29; reperibile a www.vatican.va. Sembra che l'immagine di Dio vada identificata piuttosto con il concetto della persona, che designa sia la relazione dell'essere umano, in primo luogo con Dio, sia la sua unità consistente dello strato spirituale e di quello corporale.

5 Cf. Collationes in Hexaemeron, coll. XI, 4; Opera Omnia V, p. 380b; De reductione artium ad theologiam., 8; V, 322ab.

${ }_{6} \quad$ "Intra igitur ad te et vide, quoniam mens tua amat ferventissime semetipsam; nec se posset amare, nisi se nosset; nec se nosset, nisi sui meminisset, quia nihil capimus per intelligentiam, quod non sit praesens apud nostram memoriam; et ex hoc advertis, animam tuam triplicem habere potentiam, non oculo carnis, sed oculo rationis." Itin., III, 1; V, 303b.

Si tratta della cosiddetta teoria, o analogia psicologica trinitaria usata soprattutto da Agostino. Cf. ad esempio L. Karfíková, "Lidská mysl jako model Trojice podle Augustinova spisu De Trinitate IX, X a XV", in Studie z patristiky a scholastiky, L. Karfíková (Praha: Oikumene, 1997), 62-108. La teologia trinitaria contemporanea prende una posizione piuttosto critica nei confronti di questa via, perché essa suscita inevitabilmente l'impressione che Dio sia un "supersoggetto" e che il Padre, il Figlio e lo Spirito Santo siano soltanto singole facoltà o funzioni di questa in realtà unica super-persona divina. Non è fuori luogo vedervi un implicito sabellianesimo, tipico della teologia trinitaria occidentale da Agostino in poi. Va notato, però, che il Dottore Serafico usa questa analogia piuttosto nell'ambito antropologico, mentre nella sua teologia trinitaria viene posto l'accento soprattutto sulla teoria delle relazioni ipostatiche, altra teoria di Agostino che oggi riscuote molto successo, e sul primato assoluto della persona del Padre, ispirazione proveniente piuttosto dalla teologia dell'Oriente cristiano. Cf. C. V. Pospíšil, Jako v nebi tak i na zemi. Náčrt trinitární teologie, 3. ed. (Praha - Kostelní Vydří: Krystal o.p. - Karmelitánské nakladatelství, 2017), 323-363; 386-391.

7 "San Agustín asume en su teología de la imagen un vocabulario estoico, que revela una concepción jerarquizada del alma en cuyo vértice está la mens y crea un lenguaje que acaba siendo aceptado en todo Occidente medieval. De acuerdo con este vocabulario se hace una lectura de Gén 1,26: Dios ha creado a la mens a su imagen.” F. C. Blanco, Imago Dei - Aproximacion a la antropologia teologica de san Buenaventura (Murcia: Espigras, 1993), 68. 
Il concetto della mens assume, quindi, nel pensiero bonaventuriano un significato pluridimensinale, che sorpassa quello della parola "mente" così come si usa correntemente oggi, dove con la parola "mente" si designa piuttosto la capacità razionale della persona umana. ${ }^{8} \mathrm{La}$ mente che ricorda, conosce e ama è capace pure d'ammirazione e di desiderio, vale a dire degli atti affettivi. Tutti questi elementi fanno parte dell'esperienza spirituale completa, che può essere definita anche come contemplazione intuitiva. ${ }^{9}$

Negli scritti del Dottore Serafico troviamo relativamente spesso la parola $\operatorname{cor},{ }^{10}$ che funge da sinonimo della parola mens. Ne deriva il fatto che la mente rappresenta pure un centro unificante della personalità umana. ${ }^{11}$ La teologia fatta da una mente completa deve essere sia intellettuale che affettiva. Gli atti della conoscenza sono portati avanti dal desiderio della volontà, dal desiderio di arrivare più vicino al vedere e gustare quello che viene abbracciato dall'intelletto. Inoltre va notato che la concezione della mente fa della gnoseologia bonaventuriana formalmente un'applicazione del mistero trinitario. Non vale che l'amore fa l'amante più simile all'Amato? Infine va notato che la teologia fatta con tutta la mente potrebbe essere designata anche come una teologia del cuore. Data la complementarietà tra l'intelletto e la volontà (affetto), sembra giusto affermare che l'espressione "teologia affettiva" va presa come una sineddoche, nella quale con la parte movente dell'attività della mente si vuole designare la realtà molto complessa della teologia presa come sapienza.

8 Cf. CH. N. Foshee, "St. Bonaventure and Augustinian Concept of Mens," Franciscan Studies 27 (1967): 163-175, qui p. 168; E. Cousins, "Introduction", in Bonaventure. The Soul's Journey into God. The Tree of Life. The Life of Saint Francis, E. Cousins (New York - Ramsey - Toronto: Paulist Press, 1978), 1-57, qui p. 21.

9 Cf. Itin., VI, 3; V, p. 311b; Itin., II, 5; V, p. 300b; Itin., prol. 4; V, $296 a$ b.

10 “... nullus potest effici beatus, nisi supra semetipsum ascendat, non ascensu corporali, sed cordiali." Itin., I, 1; V, 296b.

Lo stesso uso scambievole delle parole cor e mens incontriamo pure nelle opere di Tommaso d'Aquino. Cf. O. H. Pesch, Tommaso d'Aquino. Limiti e grandezza della teologia mediovale. Una introduzione (Brescia: Queriniana, 1994), 127.

11 La personalità, l'esperienza psichica del proprio io e delle sue facoltà conoscitive, volitive e affettive, non va scambiata per persona. Se la persona significa l'identità che non cambia, la personalità cresce, si edifica o decade, e pertanto non è senza cambiamenti. Le opinioni differenti degli interpreti dimostrano come è difficile cogliere il rapporto esatto tra le categorie della mente e della persona: "Mens reclama al espíritu humano en su totalidad o la función más eminente de éste.” F. C. Blanco, Imago Dei, 68; cf. E. Cousins, "Introduction," 21; Secondo G. Iammarrone, "Il posto e la funzione di Gesù Cristo nell'ascesa dell'uomo a Dio,“ Studi Francescani 85 (1988), p. 286, il concetto di mente s'identifica con l'io umano: "Il soggetto dell'ascesa (pellegrinaggio, transito) è uno e il medesimo, l'anima, ovvero, in termini moderni, l'io umano..." 
La sapienza secondo Bonaventura prima di tutto orienta tutte le cose verso la fine ultima, verso Dio. In questo senso poi non è tanto difficile identificare la sapienza con la funzione salvifica della croce che riconduce tutto al Padre.

Sapientia est habitus nobilissimus mentis quo sublevatur anima rationalis ad comprehendendum alta, ad speculandum arcana, ad degustandum suavia, ad complexandum aeterna. Apostolus hoc videtur innuere cum dicit: Ut possitis comprehendere cum omnibus sanctis quae sit latitudo et longitudo et sublimitas et profundum, scire etiam supereminentem etc. Si igitur haec est vera definitio sapentiae et lignum crucis est lignum per quod deducimur ad comprehendendum alta, ad speculandum arcana etc., lignum crucis est lignum sapientiae et ideo summe amandum est. Et hoc habetur in Apocalypsi: Beati qui lavant stolas suas in sanguine Agni, qui habent potestatem in ligno vitae ut sic per portas ingrediantur civitatem. ${ }^{12}$

Le parole comprehendere e speculandum indicano la conoscenza, e perciò rappresentano la potenza intellettuale della mente, invece le parole degustandum e complexandum si delineano con il desiderio della volontà. La sapienza cioè dice sia l'affetto che l'intelletto, come dimostra la seguente citazione: “... et hic habitus dicitur sapientia, quae simul dicit cognitionem et affectum: Sapientia enim doctrinae est secundum nomen eius, Ecclesiastici sexto."13

Adesso bisogna trovare un testo dove il nostro maestro confermerebbe quello che abbimo visto finora, più precisamente un testo in cui il Serafico direbbe espressamente che la teologia è fondamentalmente una sapienza, cioè l'attività sia dell'intelletto che della volontà in forma dell'affetto.

\section{La teologia come sapienza della croce (Sent. I, prooem., q. III, resp.; I, 13)}

Tale testo si trova in un altro punto cruciale dell'opera bonaventuriana, cioè nell'introduzione a tutto il monumentale Commento alle Sentenze. Proprio nella terza questione della parte introduttiva Bonaventura si domanda se la teologia è piuttosto una scienza speculativa oppure pratica.

12 De S. Andrea Sermo I Coll., ed. J. G. Bougerol, Sermons De diversis (Paris: Cerf 1993, n. 34, 5; p. 451; Op. Om. IX, pp. 468b-469a. Il testo nelle due edizioni critiche è identico.

13 Sent. I, prooem., q. III, resp.; I, $13 \mathrm{~b}$. 
La paternità bonaventuriana dell'introduzione a tutto il Commento alle Sentenze è un dato sicuro e indiscusso. ${ }^{14}$ Siccome la più lunga redazione, edita nell' Opera Omnia $\mathrm{I},{ }^{15}$ potrebbe rappresentare un emendamento della prima redazione, edita da Delorme, ${ }^{16}$ nel corso del nostro lavoro diamo preferenza alla prima versione menzionata. Inoltre va notato che l'introduzione stessa, che precede le questioni introduttive, rappresenta soltanto il contesto prossimo dell'oggetto diretto del nostro interesse il quale è appunto il respondeo della terza questione introduttiva, edito esclusivamente nell'Opera Omnia I.

Le numerose osservazioni riguardanti l'incarnazione, la croce e il mistero della redenzione fanno capire che questa introduzione è stata scritta, con molta probabilità, dopo la conclusione della stesura del terzo libro ${ }^{17}$ cioè alla fine di tutto il lavoro sul Commento alle Sentenze nell'anno $1252 .{ }^{18}$ Inoltre, la prima parte dell'introduzione porta i tratti evidenti del sermone universitario, e perciò appare come programma di un teologo che, dopo aver concluso la sua formazione scientifica, sta per diventare maestro all'università. ${ }^{19}$

Nella terza questione il Dottore Serafico cerca di rispondere alla domanda se lo scopo della teologia sia quello teorico (contemplationis gratia) oppure quello pratico (ut boni fiamus). ${ }^{20}$ La risposta offerta da Bonaventura non è proprio di facile interpretazione, perché nel testo incontriamo un'inclinazione a comunicare un senso nascosto tra le righe, che consiste nell'uso implicito del paradigma della cosiddetta croce intelligibile. ${ }^{21}$

14 Cf. B. Distelbrink, Bonaventurae scripta: authentica dubbia vel spuri (Roma: Istituto Storico Cappucini 1975, p. 6.

15 Cf. Sent. I, prooem., q. III, resp.; I, 13ab.

16 Cf. S. Bonaventurae, Collationes in Hexaëmeron et Bonaventuriana quaedam selecta, ed. F. Delorme (Firenze: Quaracchi, 1934), 279-283.

17 Bonaventura ha commentato i singoli libri nell'ordine seguente: I, IV, II, III. Cf. I. Brady, The Edition of the "Opera omnia"? of Saint Bonaventure (1882-1902) (Grottaferrata: Quaracchi, 1977), 133-134. Nel lavoro citato Brady ha precisato l'opinione precedente, secondo la quale i libri dovrebbero essere commentati nell'ordine: I, II, IV, III. Cf. B. Distelbrink, "De ordine chronologico IV Librorum Commentarii in Sententias S. Bonaventurae," Collectanea Franciscana 41 (1971): 288-314. In ogni caso, gli esperti concordano sul fatto che il terzo libro è stato commentato per ultimo.

18 Cf. B. Distelbrink, Bonaventurae scr., 5.

19 Cf. A. Ménard, "Une leçon inaugurale de Bonaventure. Le prooemium du Livre des Sentences," Études Franciscaines 21 (1971): 273-289.

20 "Tertio quaeritur de causa finali. Et cum dictum sit, quod liber iste est ad revelandum abscondita, quaeritur, utrum opus hoc sit contemplationis gratia, vel ut boni fiamus." Sent. I, prooem., q. III; I, p. 12a.

21 La croce intelligibile rappresenta un paradigma geometrico che permette a Bonaventura di chiarire certi aspetti del mistero. Cf. C. V. Pospíšil, La salvezza 
Nel titolo, preposto dagli editori al respondeo, la teologia viene designata come un abito affettivo. ${ }^{22}$ Proprio qui, a nostro avviso, si trova una delle sorgenti del malinteso da parte di coloro che non comprendono bene il complesso significato della parola "affetto" nel pensiero bonaventuriano, come abbiamo detto sopra. Il Dottore Serafico stesso, però, dice con tanta chiarezza che la teologia è soprattutto la sapienza, che possiede sia la dimensione intellettuale, che quella affettiva, come abbiamo visto nella citazione esposta sopra. ${ }^{23}$

Bonaventura non può non mettere in rilievo la dimensione intellettuale della teologia, ${ }^{24}$ perché altrimenti tutto l'enorme sforzo intellettuale del commentatore delle Sentenze non avrebbe nessun senso. Dato che la teologia appartiene alle scienze, perché altrimenti non potrebbe essere insegnata all'università, bisogna cominciare proprio dall'intelletto che viene perfezionato mediante lo sforzo del ricercatore. ${ }^{25}$

Nam si consideremus intellectum in se, sic est proprie speculativus et perficitur ab habitu, qui est contemplationis gratia, qui dicitur scientia speculativa. Si autem consideremus ipsum ut natum extendi ad opus, sic perficitur ab habitu, qui est ut boni fiamus; et hic est scientia practica sive moralis. Si autem medio modo consideretur ut natus extendi ad affectum, sic perficitur ab habitu medio inter pure speculativum et practicum, qui complectitur utrumque; et hic habitus dicitur sapientia, quae simul dicit cognitionem et affectum. ${ }^{26}$

Siccome l'intelletto viene perfezionato da tre abiti diversi e si protende nelle tre direzioni corrispondenti, e siccome una di queste direzioni si trova in mezzo alle altre due, non sembra affatto fuori luogo ricostruire lo schema geometrico implicito al pensiero del Dottore Serafico. Dato che la contemplazione ha nel periodo parigino dell'opera del nostro autore sempre la connotazione di ascesa, essa dovrebbe portare l'intelletto in alto. L'abito pratico dell'agire morale si protende

dell'uomo e la teologia della croce di Bonaventura da Bognoregio (Udine: Paolo Gasprari editore, 2010), 167-190.

22 "Scientia theologica est habitus affectivus ..." Sent. I, prooem, q. III; I, 13a.

23 Cf. Sent. I, prooem., q. III, resp.; I, 13 b.

24 Il fatto che Bonaventura certamente non sottovaluta il lato intellettuale del lavoro teologico viene documentato dal seguente pensiero: "Ex praedictis ergo apparet, quo ordine et quo auctore pervenitur ad sapientiam. Ordo enim est, ut inchoetur a stabilitate fidei et procedatur per serenitatem rationis, ut perveniatur ad suavitatem contemplationis ..." Sermo Christus unus omnium magister, 15; V, 571b.

25 "Ad intelligentiam praedictorum notandum est, quod perfectibile a scientia est intellectus noster." Sent. I, prooem., q. III, resp.; I, 13a.

26

Sent. I, prooem., q. III, resp.; I, 13ab. 
verso il mondo esteriore in cui viviamo, quindi verso il basso. In mezzo a queste due direzioni si trova quella orizzontale, che si potrebbe dire linea dell'interiorità della vita psichica, linea che porta l'intelletto verso l'affetto. Esattamente nel punto centrale dell'asse orizzontale si trova la sapienza, che abbraccia sia l'intelletto che l'affetto e, nello stesso tempo, costituisce pure il centro dell'asse verticale, perché rappresenta la mediazione tra la "contemplationis gratia", la teoria, l'intravedere le idee, l'ascesa mistica da una parte e l'“ut boni fiamus", cioè il giudizio morale e pratico, il nostro modo di agire dall'altra. La sapienza, trovandosi nel punto centrale della croce intelligibile, vale a dire nell'intersezione dell'asse orizzontale con quello verticale, ${ }^{27}$ unifica e armonizza i quattro elementi costitutivi del corretto pensare e vivere teologico; nello stesso tempo, essendo un dono divino, li sorpassa e corona dando loro il senso trascendente, che consiste nel rapporto diretto con il fine ultimo della vita umana e di tutto il mondo creato. Per tutti questi motivi dovrebbe essere chiaro perché il secondo nome della dottrina trasmessa nel libro delle Sentenze è proprio "la Sapienza".

Un argomento a favore di questa nostra interpretazione consiste nel fatto che la teologia e la Sacra Scrittura erano per Bonaventura, soprattutto negli ultimi anni del suo lavoro all'università parigina, i due concetti che, avendo la stessa funzione, cioè di far entrare il Verbo divino nella mente umana, si potevano reciprocamente scambiare. Se nel prologo al Breviloquium la croce intelligibile rappresenta l'unica vera chiave ermeneutica, non dovrebbe allora lo stesso valere pure per la dottrina contenuta nel suo Commento alle Sentenze? La teologia, dovendo servire all'autodonazione divina all'uomo, non dovrebbe essere un'icona del Crocifisso? Alla domanda il Dottore Serafico risponde richiamando espressamente la morte di Cristo:

Talis est cognitio tradita in hoc libro. Nam cognitio haec iuvat fidem, et fides sic est in intellectu, ut, quantum est de sui ratione, nata sit movere affectum. Et hoc patet. Nam haec cognitio, quod Christus pro nobis mortuus, et consimiles, nisi sit homo peccator et durus, movet ad amorem ...28

\footnotetext{
27 Questa collocazione della sapienza nel centro della croce intelligibile corrisponde esattamente a quello che abbiamo visto durante la nostra analisi di questo paradigma nella prima conferenza dell'Hexaëmeron. Cf. Hex., coll. I, n. 24; V, 333b. 
La scelta dell'esempio usato non sembra affatto casuale, perché nell'introduzione al terzo libro del Commento alle Sentenze leggiamo che tutta la dottrina trasmessa nei quattro libri delle Sentenze tratta principalmente del mistero della nostra redenzione. ${ }^{29}$ Ne deriva che il mistero della redenzione rappresenta per il Dottore Serafico pure un principio architettonico, cioè quello centrale e unificante di tutta la sua riflessione teologica. La teologia in quanto la partecipazione della nostra mente alla Sapienza di Dio assume, quindi, una forma della croce e persino del Crocifisso.

Siccome questa nostra interpretazione della terza questione introduttiva al Commento alle Sentenze è completamente nuova e siccome essa ci permette di vedere tutto il pensiero teologico del Dottore Serafico in una luce nuova, cioè come una teologia sapienziale della croce, conviene offrire ancora un argomento a suo favore, che consiste nel passo biblico richiamato da Bonaventura nel brano che stiamo esaminando, perché il versetto Sir 6, 23 si trova in un altro testo, ben noto a Bonaventura, e forse anche ai suoi studenti, che il nostro autore cita in altra opera:

Dico quod in ligno crucis Christi possumus invenire, primo, sapientiam salutarem ad nos illuminandum et docendum ... Vae illis qui toto tempore vitae suae student in logica, physica, vel in decretis, et nihil saporis in ista scientia inveniunt. Si in ligno crucis Christi studerent, scientiam salutarem ibi invenirent. ... Ista disponit hominem ad intelligentiam, conservat in eo memoriam, ordinat ad vitam aeternam. Ideo beatus Bernardus $\mathrm{s}^{30} \mathrm{de}$ hoc ligno ita ait: Cum sapientia secundum nomen suum dicta sit saporosa scientia, in ligno crucis stude, si vis hunc saporem sentire; dat enim visum caecis, sanitatem infirmis, vitam mortuis. ${ }^{31}$

29 "Nam in primo libro agitur de reparationis auctore, utpote de beata Trinitate. In secundo vero agitur de ipso reparabili, utpote de homine cadente a statu conditionis innocentiae. In tertio agitur de persona Redemptoris, utpote de Christo, Deo et homine. In quarto vero agitur de hominis reparati salute, quae quidem consistit in expiatione culpae et amotione omnis miseriae. Et sic patet, quomodo iste totalis liber versatur circa nostrae reparationis mysterium explicandum ..." Sent. III, prooem.; III, p. 1b-2a. L'espressione Totalis liber significa tutto il Commento alle Sentenze. D'altronde entrambe le introduzioni, cioè quella al terzo libro e quella al primo libro devono essere state scritte in un breve arco di tempo, perché le introduzioni si scrivono quando tutto il resto del libro già esiste e perché Bonaventura ha commentato il terzo libro per ultimo.

30 In realtà si tratta piuttosto di un pensiero proveniente da Cassiodoro.

31 Dom. II post Pascha Sermo V, Bougerol, DeDiv, n. 24, 7; 337-338; Op. Om. IX, p. 304b. Il testo nelle diverse edizioni critiche sostanzialmente corrisponde. Per quanto riguarda 
Non solo la citazione di Bernardo, o piuttosto Cassiodoro, legata al versetto Sir 6, 23, bensì le parole di Bonaventura dicono chiaramente che la teologia vera deve essere sapienza della croce, e perciò non si può più dubitare circa il senso contenuto nella conclusione della terza questione introduttiva al Commento alle Sentenze. Infatti, la croce è la sorgente di ogni vera sapienza proveniente da Dio. ${ }^{32}$

Alla domanda, per quale motivo Bonaventura non sia stato più esplicito proprio in questo passo così importante, si può rispondere assai facilmente. Infatti, il nascondersi della Sapienza divina sotto l'involucro del senso letterario apparente a tutti è una caratteristica del Verbo, sia nella Sacra Scrittura che nell'evento dell'incarnazione; ${ }^{33}$ il Dottore Serafico, nella sua ricerca appassionata della vera Sapienza divina, ha cercato di imitarla perfino nel modo di esprimere i suoi misteri, perché colui che porterà sempre nel cuore il ricordo della passione del Signore sicuramente saprà scendere nelle profondità dell'intelligenza nascosta e porterà alla luce quello che voleva comunicargli velatamente il grande maestro della sapienza della croce. Questa sapienza della croce, però, deve rimanere chiusa a coloro, che s'interessano solo della raccolta d'informazioni. ${ }^{34} \mathrm{Si}$ vede che Bonaventura non andrebbe sicuramente d'accordo con una teologia puramente intellettualistica staccata dall'impegno spirituale ed esistenziale.

\section{Attualità della concezione bonaventuriana della teologia}

Innanzi tutto la teologia "affettiva" di Bonaventura va presentata come una teologia sapienziale, una teologia del cuore, perché coinvolge non solo l'intelletto, ma pure le forze affettive della persona umana. L'espressione "una teologia affettiva" va presa come una sineddoche, la quale designa attraverso movente affettivo dell'attività sapienziale.

La teologia intesa come puro sforzo razionale, la teologia secondo le misure della cosiddetta scientometria che oggi regge l'attività di molti universitari, putroppo pure di tanti colleghi dalle facoltà teologiche nella Reppublica Ceca, rischia di non essere conforme allo spirito della

alcuni dettagli, abbiamo dato precedenza all'edizione nell'Opera Omnia perché questa versione ci pare più logica.

32

33

Cf. Breviloquium., prol., $\$ 4$; V, p. $206 \mathrm{~b}$.

34 In questo senso dobbiamo, purtroppo, valutare l'interpretazione contenuta in: I. Biffi, Figure medievali della teologia (Milano: Jaca Book, 1992), 97-98. 
rivelazione, la quale non è soltanto un'informazione, ma l'autodonazione di Dio con lo scopo della nostra profonda trasformazione. Un altro rischio riguarda la sempre più stretta specializzazione che perde di vista l'insieme della teologia e soprattutto il suo scopo primariamente salvifico. Per questo motivo la Commissione Teologica Internazionale nel suo documento "Theology Today" ammonisce tutti noi, affinché non perdiamo di vista la dimensione sapienziale del nostro sforzo. ${ }^{55}$ A questa voce dal punto di vista professionale autorevole si aggiunge anche il nostro sforzo presentato in questo articolo. La teologia deve essere sicuramente scientifica, ma non andare oltre significherebbe restare a metà strada. La teologia vera, la teologia come aiuto, come indicazione della strada che porta verso la salvezza, ${ }^{36}$ non può essere fatta senza il cuore, senza l'affetto, senza l'aspirazione di comunicare in qualche modo la propria esperienza spirituale.

Infine, ripensando la figura della teologia bonaventuriana espressa nella terza questione dell'introduzione al Commento alle Sentenze, ci rendiamo conto che lo scopo di tutto lo sforzo di un dogmatico deve mirare alla prassi (ut boni fiamus), apportando i motivi teoretici e offrendo il perché di queste esigenze del Vangelo. Il senso ultimo di tutti i dogmi non è semplicemente una dottrina, ma la vita. Se lo studente capisce il significato del dogma nella propria vita spirituale, comprende il vero senso dell'enunciato dogmatico; comprende che questa verità è la nostra vita espressa nei termini a prima vista teorici. In questo modo avviene una spirituale quasiverificazione, dalla quale può scaturire una teologia come testimonianza.

Catholic Theological Faculty, Charles University Thákurova 3 16000 Praha 6 E-mail: ctirad.pospisil@ktf.cuni.cz

35 Cf. Commissione Teologica Internazionale, Theology Today, documento dall'anno 2012, in Dokumenty Mezinárodni teologické komise 1969-2017 a nékteré dalši texty Papežské biblické komise a Kongregace pro nauku víry, eds. C. V. Pospíšil - E. Krumpolc (Olomouc: Vydavatelství Univerzity Palackého, 2017), 879-931; qui n. 60, 909-910; n. 67,913 ; n. 82, 921.

36 Non dimentichiamo che il compito principale di tutta la teologia consiste nel condurci "all'albero della vita," per usare le parole del Dottore Serafico. Cf. Hex., coll. I, 38; V, p. 335; Hex., coll. XIX, 1; V, p. 420; Hex., coll. XXIII, 31; V, 449b. 